\title{
Disfunção craniocervicomandibular ascendente de origem postural: relato de caso
}

Ascending craniocervical mandibular disorder of postural origin: case report

Disfunción craneocervicomandibular ascendente de origen postural: reporte de caso

Bárbara Nathalia Marques de LIMA

Lioney Nobre CABRAL

Escola de Ciências da Saúde, Universidade do Estado do Amazonas (ESA-UEA), 69065.001, Manaus-AM, Brasil

\section{Resumo}

A disfunção craniocervicomandibular ascendente acontece quando alterações situadas abaixo do complexo craniomandibular são os fatores desencadeantes de uma desordem temporomandibular. Os sinais e sintomas mais comumente encontrados nessa disfunção são: dor localizada nos músculos mastigatórios ou na região da articulação temporomandibular, cefaléia, ruídos articulares e limitações dos movimentos mandibulares. O objetivo do presente estudo é relatar um caso de disfunção craniocervicomandibular ascendente causada por uma condição postural inadequada decorrente de maus hábitos ergonômicos. Um paciente procurou a Policlínica Odontológica da Universidade do Estado do Amazonas queixando-se de dor intermitente na região lateral da face associada à otalgia. Ao exame clínico, observou-se estalidos na articulação temporomandibular, presença de pontos-gatilhos nos músculos cervicais e retificação cervical, sendo assim diagnosticado com a disfunção, causada pela alteração postural. Após o diagnóstico, optou-se por uma modalidade de tratamento conservadora baseada na realização de exercícios fisioterapêuticos associados ao uso de uma placa estabilizadora miorrelaxante. Após um mês de tratamento, o paciente já apresentou um estado postural mais adequado, sem sintomatologia dolorosa. Após três anos de proservação, o paciente manteve o quadro estabilizado, sem queixas de dor. Concluímos que o correto diagnóstico da condição é a chave para a escolha e o sucesso do tratamento, sendo este realizado através de uma avaliação minuciosa e integral do indivíduo durante o exame clínico.

Descritores: Síndrome da Disfunção da Articulação Temporomandibular; Postura; Dor Facial.

\section{Abstract}

Ascending craniocervical mandibular disorder occurs when disturbance below the craniomandibular complex are the triggering factors of a temporomandibular disorder. The signs and symptons most commonly found in this dysfunction are: located pain in masticatory muscles or in the region of temporomandibular joint, headache, joint noises and limited mandibular movements. The aim of this study was to report a case of ascending craniocervical mandibular disorder caused by an inadequate postural condition due to poor ergonomic habits. Patient sought the Dental Polyclinic of the Amazonas State University complaining about intermittent pain in the lateral region of the face associated with earache. The clinical examination revealed a clicking on the temporomandibular joint, presence of trigger points in cervical muscles and cervical rectification, being thus diagnosed with the dysfunction caused by the postural alteration. After diagnosis, a conservative treatment modality was adopted based on physical therapy exercises, and after that a stabilizer splint to be used in association with the exercises was prepared and installed. After a month of treatment, the patient was already in a more adequate postural state, presenting no painful symptomatology. After three years of proservation, the patient remained stable, with no complaints of pain. Thus, we conclued that the key to the choice and success of treatment is the correct diagnosis of the condition that is performed through a assiduos and integral evaluation of the individual during the clinical examination.

Descriptors: Temporomandibular Joint Dysfunction Syndrome; Posture; Facial Pain.

\section{Resumen}

La disfunción craneocervicomandibular ascendente ocurre cuando los cambios debajo del complejo craneomandibular son los factores desencadenantes de un trastorno temporomandibular. Los signos y síntomas más comúnmente encontrados en esta disfunción son: dolor localizado en los músculos masticatorios o en la región de la articulación temporomandibular, dolor de cabeza, ruidos articulares y limitaciones de los movimientos mandibulares. El objetivo del presente estudio es informar un caso de disfunción craneocervicomandibular ascendente causada por una condición postural inadecuada como resultado de malos hábitos ergonómicos. Un paciente buscó a la Clínica Dental de la Universidad Estatal de Amazonas quejándose de dolor intermitente en la región lateral de la cara asociada con otalgia. El examen clínico reveló clics de la articulación temporomandibular, puntos gatillo en los músculos cervicales y rectificación cervical, diagnosticada con disfunción causada por alteración postural. Después del diagnóstico, se eligió una modalidad de tratamiento conservador, basada en ejercicios de fisioterapia asociados con el uso de una placa estabilizadora miorrelajante. Después de un mes de tratamiento, el paciente ya presentaba un estado postural más adecuado, sin síntomas dolorosos. Después de tres años de conservación, el paciente permaneció estable, sin quejas de dolor. Llegamos a la conclusión de que el diagnóstico correcto de la condición es la clave para la elección y el éxito del tratamiento, que se realiza a través de una evaluación exhaustiva del individuo durante el examen clínico.

Descriptores: Síndrome de la Disfunción de Articulación Temporomandibular; Postura; Dolor Facial.

INTRODUÇÃO

A articulação temporomandibular (ATM) faz conexões musculares e ligamentares na região cervical, formando um complexo funcional chamado de sistema craniocervicomandibular (sistema manducatório). Este é considerado uma unidade biomecânica composta pela mandíbula, crânio, osso hióide, músculos supra e infra-hióideos e coluna cervical ${ }^{1}$.

Funcionalmente, a coluna cervical, ATM e as articulações entre os dentes estão intimamente relacionadas e a anormalidade funcional ou a má posição de uma delas pode afetar a função ou a posição das outras. Como exemplar podemos citar a alteração na posição da cabeça, que modifica a posição mandibular, acometendo, assim, a oclusão; e o equilíbrio entre os flexores e os extensores da cabeça e do pescoço pode ser afetado pelos músculos da mastigação e pelos supra e infra-hióideos, logo, a disfunção nos músculos da mastigação ou nos músculos cervicais pode, facilmente, alterar este equilíbrio. Assim, as complexas interações anatômicas e biomecânicas entre o sistema 
estomatognático e a área de cabeça e pescoço permitem que haja uma relação entre disfunção temporomandibular (DTM) e postura ${ }^{2,3}$.

A postura de cada indivíduo é determinada por cadeias musculares, fáscias, ligamentos e estruturas ósseas, que possuem solução de contiguidade, sendo interdependentes entre si, abrangendo todo o organismo. Para mantê-la é necessário um complexo sistema neuromuscular integrado com vários proprioceptores em músculos, tendões, articulações e receptores vestibulares e visuais de áreas motoras corticais e subcorticais. Alguns fatores podem influenciar a postura corporal e promover alterações, como: estados de humor, ansiedade, posição da cabeça e pescoço, funções orais (respiração, deglutição), sistema oculomotor e visual e alterações na orelha interna ${ }^{3-5}$.

Uma alteração local repercute em compensações por todo o corpo em forma de cascata, podendo esta ser de forma ascendente ou descendente. Uma deformação ascendente ocorre quando um desequilíbrio no segmento inferior do corpo produz compensações em segmentos superiores, como o posicionamento anterior da cabeça, provocando distúrbios de posicionamento e funcionamento mandibular, tensionando a musculatura mastigatória, causando DTM. Já uma deformação descendente ocorre quando um desequilíbrio no segmento superior do corpo produz compensações em segmentos inferiores, como exemplar tem-se a hiperatividade dos músculos da mastigação que pode interferir na atividade dos músculos de contra apoio (esternocleidomastóideo e trapézio) levando ao encurtamento dos músculos posteriores do pescoço e alongamento dos músculos anteriores, resultando em uma projeção anterior da cabeça ${ }^{4}$.

A posição anterior da cabeça, alteração postural mais prevalente, aumenta a extensão do osso occipital sobre a primeira vértebra, atlas, e esta sobre o áxis, o que compromete a mobilidade, diminuindo a lordose cervical fisiológica, aumentando a cifose torácica com elevação e protração dos ombros. Há um encurtamento dos músculos extensores cervicais posteriores (suboccipitais, semiespinal, esplênio), supra-hióideos e esternocleidomastóideo; alongamento dos infrahióideos e elevação do osso hióide, com reposicionamento mandibular e hiperatividade dos músculos da mastigação (temporal e masseter), promovendo uma força de elevação e retrusão que atua na mandíbula, diminuindo o espaço funcional livre. Além disso, a tensão aumentada dos músculos mastigatórios e suprahióideos causa, também, uma elevação e retrusão mandibular. Assim, a posição da cabeça para frente pode exercer influência sobre a posição, bem como sobre o movimento da mandíbula e está frequentemente relacionada à dor no pescoço, devido à sobrecarga dos músculos cervicais posteriores realizada na tentativa de manter o equilíbrio da cabeça sobre a coluna ${ }^{6,7}$.

A disfunção craniocervicomandibular é uma condição comum, representada por um conjunto de sinais e sintomas que envolvem sensibilidade e/ou dor à palpação dos músculos da mastigação e cervicais, limitação da amplitude dos movimentos fisiológicos, desarranjos internos da ATM, ruídos articulares e alterações craniocervicais importantes ${ }^{7}$.

$$
\text { Os fatores envolvidos }
$$
desencadeamento desses distúrbios são: lesões degenerativas ou traumáticas da ATM, interferência oclusal, problemas esqueléticos, hiperatividade do músculo temporal e masseter, hábitos parafuncionais e fatores psicológicos, como estresse. Além disso, as anormalidades posturais foram postuladas por terem uma influência no desenvolvimento e perpetuação da disfunção ${ }^{8}$.

As modalidades de tratamento incluem: educação do paciente e autocuidado; modificação do comportamento (técnicas de relaxamento); medicamentos; placas oclusais estabilizadoras; fisioterapia e cirurgias articulares. O profissional deve avaliar cada terapêutica de acordo com a alteração apresentada pelo paciente, observando presença de desconforto, dor, limitação funcional e, a partir disso, analisar as indicações e contraindicações de cada modalidade ${ }^{9,10}$.

As placas oclusais são utilizadas para promover uma posição articular ortopedicamente mais estável e uma oclusão funcional ótima, que reorganiza a atividade reflexa neuromuscular anormal enquanto propicia uma função muscular mais adequada. Além disso, também são indicadas como barreira física de proteção para as estruturas de suporte, nos pacientes que apresentam bruxismo. É uma modalidade largamente utilizada, pois sua confecção é simples, de baixo custo, é reversível e apresenta resultados positivos quanto a melhora no quadro de disfunção ${ }^{9}$.

O tratamento fisioterapêutico tem como objetivos, aliviar a dor músculo-esquelética, reeducando o sistema neuromuscular para reestabelecer a posição de repouso mandibular e coordenação muscular; reduzir a inflamação; 
melhorar a postura; e restaurar a função motora oral, além de melhorar a qualidade de vida. Sendo considerada uma terapia relativamente simples, não-invasiva e de baixo custo, baseiase, de uma forma geral, na realização de exercícios, massagens, alongamentos, terapia de liberação posicional, ultrassom e lase ${ }^{11}$.

$\mathrm{O}$ objetivo do presente estudo é relatar um caso clínico de disfunção craniocervicomandibular ascendente causada por uma condição postural inadequada, decorrente de maus hábitos ergonômicos, cujo tratamento conservador fisioterapêutico foi instituído.

\section{CASO CLÍNICO}

Paciente do gênero masculino, 18 anos de idade, leucoderma, natural da cidade de Manaus - Amazonas, foi encaminhado por um cirurgião bucomaxilofacial à Policlínica Odontológica da Universidade do Estado do Amazonas (UEA), para avaliação clínica, com hipótese diagnóstica de disfunção temporomandibular (DTM). Durante a anamnese, o paciente relatou dor intermitente na região lateral da face há aproximadamente um ano, com presença de estalidos na região da articulação.

Ao exame clínico extraoral inicial, observou-se que o paciente apresentava uma postura craniocervical incorreta, constatada posteriormente, ser decorrente de hábitos ergonômicos inadequados (Figura 1). O paciente não apresentou limitação de abertura bucal, nem restrições mandibulares ao realizar os movimentos de lateralidade direita e esquerda. Durante a abertura, foi identificado um desvio para a direita em $19 \mathrm{~mm}$.

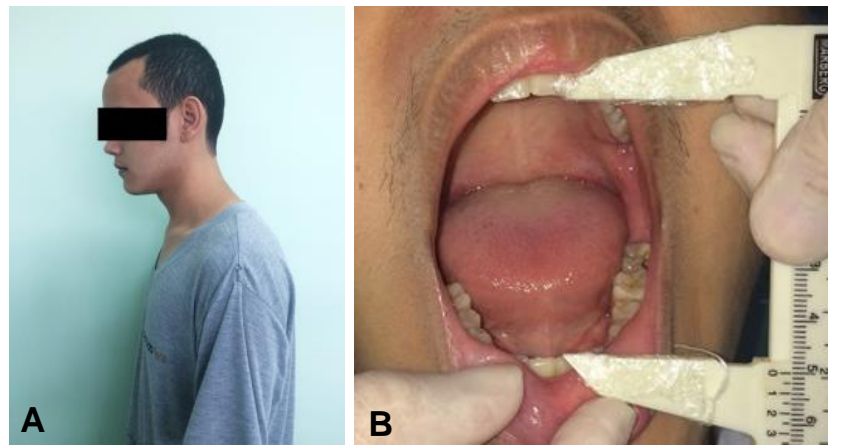

Figura 1: A) Vista lateral do paciente; B) Abertura bucal máxima $(48 \mathrm{~mm})$

Mediante a realização da palpação dos músculos da mastigação e dos músculos cervicais, o paciente relatou desconforto na porção distal e dor nas porções proximal e corpo do músculo masseter direito. Também referiu desconforto na porção clavicular do músculo esternocleidomastóideo direito (Figura 2A) e dor nas porções mastóidea e clavicular no músculo esternocleidomastóideo esquerdo (Figura 2B), regiões que continham pontos de gatilho (PsG), causando dor referida para a área temporal e pós-auricular, além de otalgia. O paciente foi então diagnosticado com disfunção craniocervicomandibular ascendente associada à síndrome dolorosa miofascial.

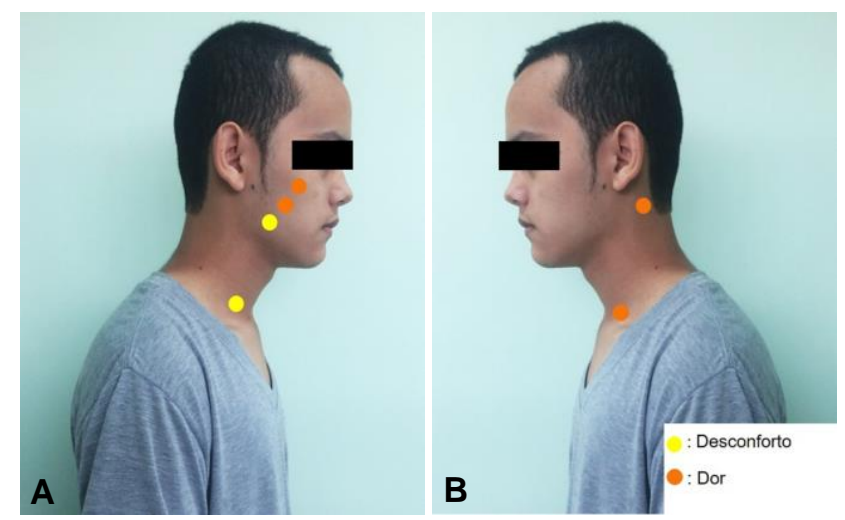

Figura 2: Localização dos PsG. A) Músculos masseter e esternocleidomastoideo direito, $\quad$ B) Músculo esternocleidomastóideo esquerdo.

Ainda na primeira sessão foram realizados procedimentos de moldagem para a obtenção dos modelos e, consequente confecção de uma placa estabilizadora miorrelaxante. O paciente foi orientado a realizar dois tipos de exercícios fisioterápicos que visam 0 alongamento da musculatura cervical. No primeiro, uma haste de madeira é posicionada entre os braços e as costas, com as mãos em palma e a cabeça bem orientada sobre a cintura escapular, segurando-a $90^{\circ} \mathrm{em}$ relação ao tronco (Figura 3). No segundo, abre os braços colocando as mãos sobre os ombros, ao mesmo tempo em que inclina a cabeça para trás, inspirando, com os cotovelos sendo deslocados sincronicamente para trás e retornando à posição anterior gradativamente, com expiração pela boca (Figura 4). Ambos realizados durante dez minutos, três vezes ao dia.

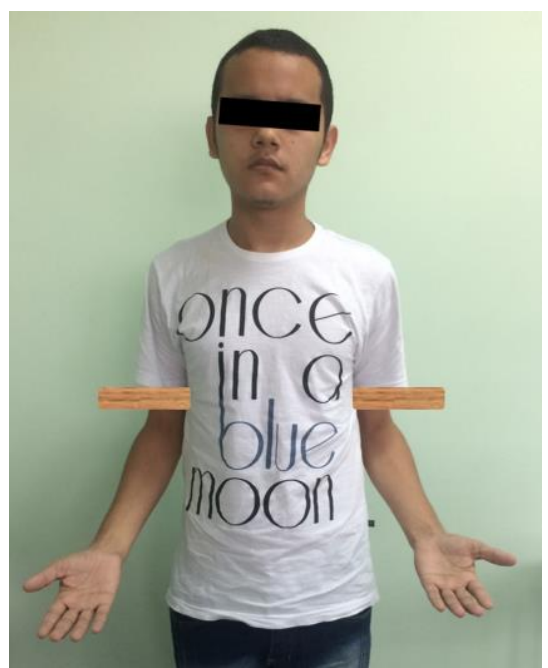

Figura 3: Exercício com haste de madeira. 


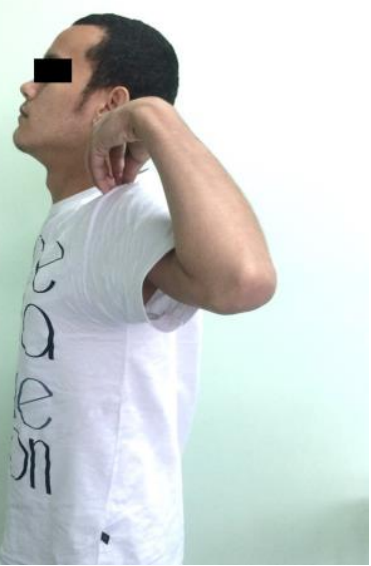

Figura 4: Exercício com inclinação da cabeça e ombros.

Após uma semana de tratamento fisioterápico, uma melhora significativa na sua condição postural já era percebida (Figura 5). Decorrido um mês do início do tratamento, o mesmo encontrava-se sem queixas álgicas e com características posturais mais adequadas (Figura 6).

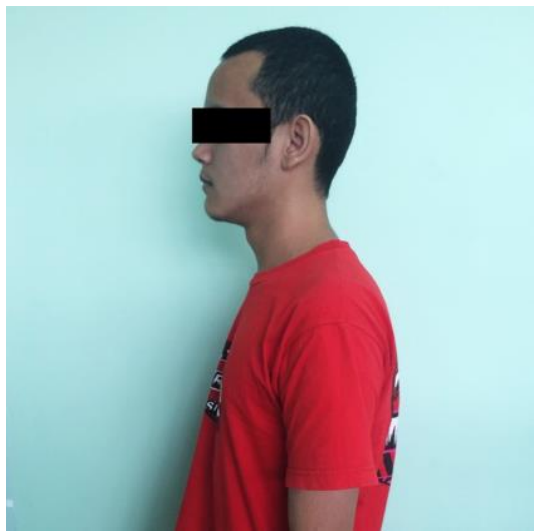

Figura 5: Paciente após 1 semana do início do tratamento.

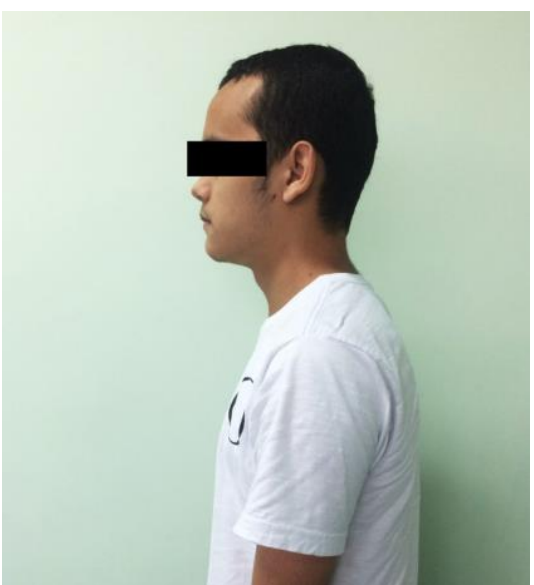

Figura 6: Paciente após 1 mês do início do tratamento.

A placa estabilizadora miorrelaxante foi instalada (Figura 7) e dois outros tipos de exercícios fisioterápicos foram prescritos, o de abertura contra resistência e o de abertura orientada. No primeiro, o paciente coloca as mãos sob o queixo gerando uma resistência contra à abertura bucal, realizando 10 repetições por 10 segundos, 3 vezes ao dia. No segundo, o paciente usa os dedos polegar e indicador para abrir e fechar a boca, de forma orientada por esses dedos, 30 vezes, contando na abertura.

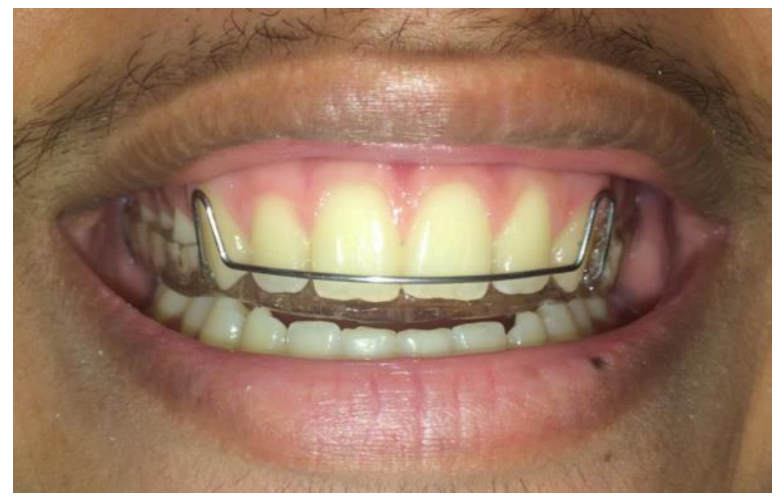

Figura 7: Instalação da placa estabilizadora miorrelaxante.

Após um ano de proservação, o paciente encontra-se com os sinais e sintomas da disfunção estabilizados, não apresentando sintomatologia dolorosa e qualquer outra queixa (Figuras 8 e 9).

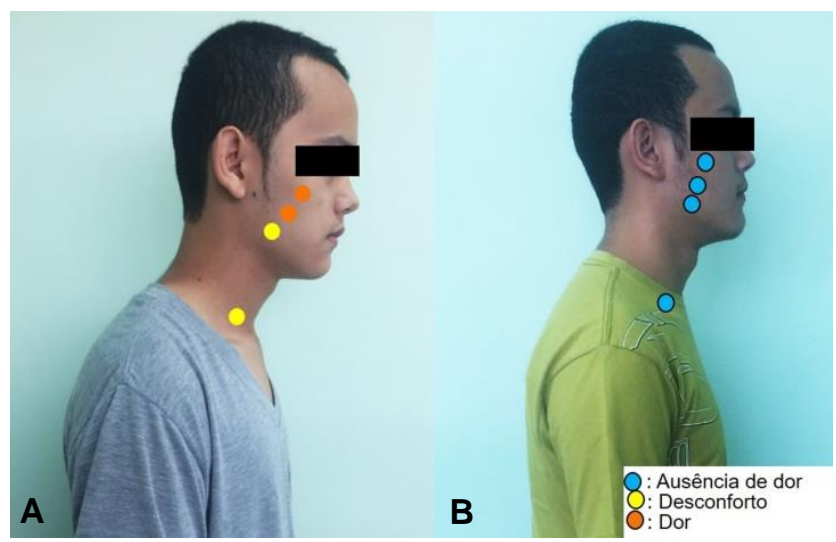

Figura 8: Evolução dos PsG do lado direito do paciente. Inicial (A). Final (B).

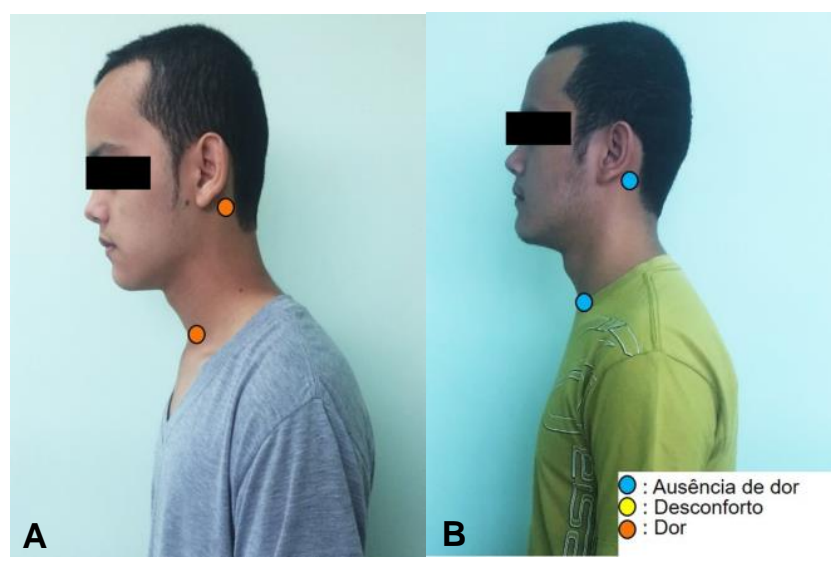

Figura 9: Evolução dos PsG do lado esquerdo do paciente. Inicial (A). Final (B).

O paciente retornou para proservação após três anos do início do tratamento, não apresentando nenhuma queixa compatível com a disfunção. Ao exame clínico extraoral, observou-se uma reorganização da lordose cervical fisiológica (Figura 10), evidenciando a importância do acompanhamento clínico a longo prazo, e o mesmo foi orientado a refazer os mesmos exercícios realizados anteriormente. 


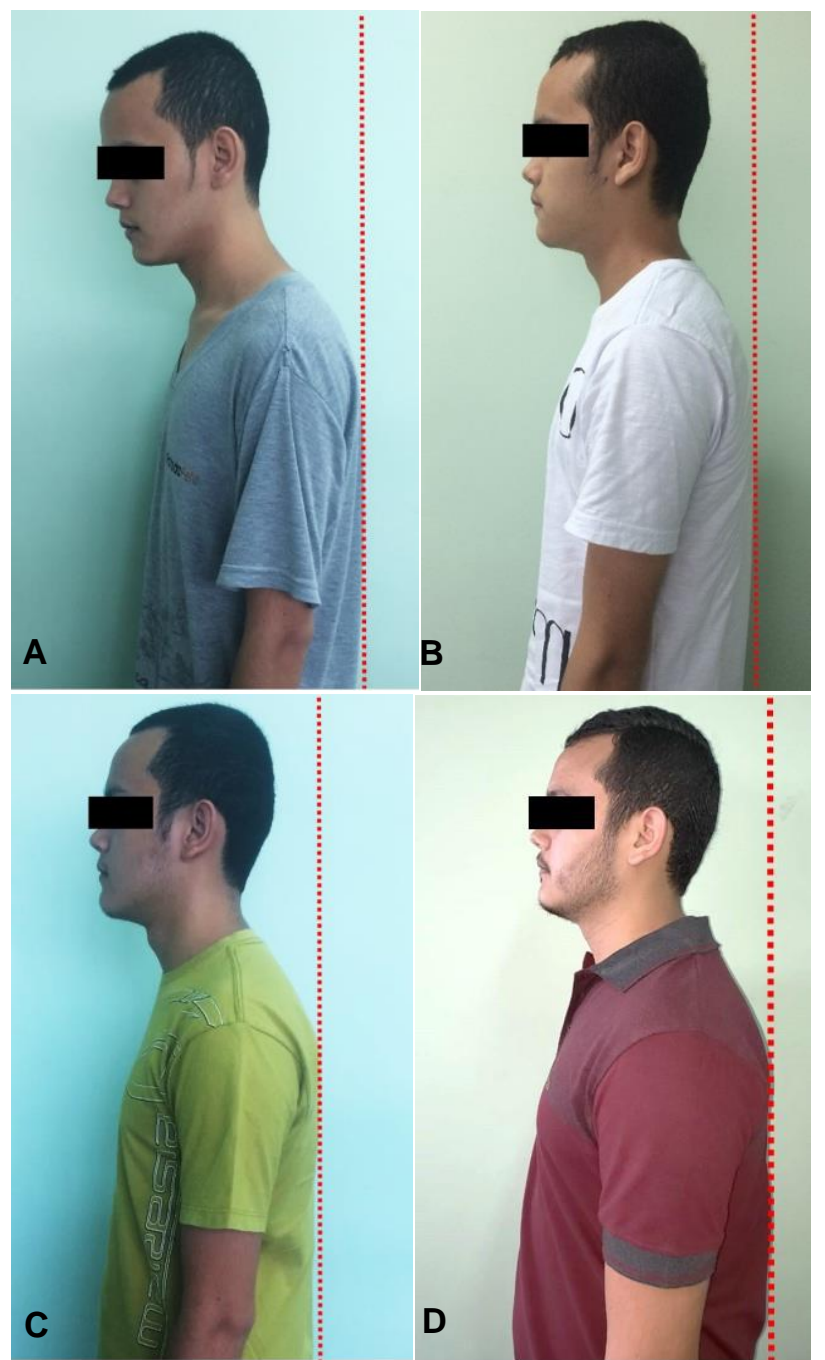

Figura 10: Quadro evolutivo do paciente. Estado postural inicial (A). Após 1 mês (B). Após 1 ano (C). Após 3 anos (D).

DISCUSSÃO

A disfunção craniocervicomandibular é uma condição crônica que afeta a região cervical, o sistema estomatognático, a articulação temporomandibular (ATM) e estruturas associadas. É de etiologia multifatorial, estando relacionada com fatores estruturais, neuromusculares, oclusais (como perdas dentárias), psicológicos (como 0 estresse), hábitos parafuncionais (como o bruxismo), lesões traumáticas ou degenerativas da ATM e alterações posturais. No presente caso, a alteração postural causada por hábitos ergonômicos inadequados, foi considerada 0 principal fator desencadeador da disfunção $0^{10-12}$.

Com relação à incidência, há concordância na literatura sobre a predominância no gênero feminino. Alguns estudos epidemiológicos mostraram uma proporção de 8 mulheres com disfunção para cada homem e isto pode estar relacionado ao fato que as mulheres possuem ligamentos mais frágeis, fatores emocionais exacerbados e uma taxa hormonal variável, que gera tensão e, consequentemente, aumento nos hábitos parafuncionais. No entanto, o caso apresentado refere-se a um paciente do gênero masculino associada a um componente físico ascendente $e^{4,13-15}$.

O paciente tinha 18 anos de idade, estando de acordo com literatura que afirma que a condição pode ocorrer em todas as faixas etárias, sendo mais prevalente entre 20 e 45 anos de idade ${ }^{3,16}$.

Os principais sinais e sintomas presentes na disfunção são: sensação de rigidez e/ou de fadiga nos músculos mastigatórios, zumbidos ou vertigens, ruídos articulares (estalidos e crepitação), alterações auditivas (sensação de diminuição de acuidade auditiva), otalgia, limitação dos movimentos e/ou desvios dos movimentos mandibulares, dor ao nível da ATM e/ou músculos mastigatórios, cefaléia, dor facial, pontos de tensão muscular (pontos de gatilho) e postura inadequada. Os sinais e sintomas apresentados pelo paciente coincidem com aqueles descritos na literatura, no entanto, o paciente não possuía limitações nos movimentos mandibulares, apresentando a abertura bucal máxima dentro dos padrões de normalidade, porém com deflexão à direita com retorno a linha média e presença de pontos de gatilho no músculo masseter ipsilateral ${ }^{2,12-14}$.

Muitos pacientes que são portadores de disfunção da articulação temporomandibular apresentam alterações importantes na postura, estas como causa ou consequências da condição, sendo a principal delas 0 posicionamento anteriorizado da cabeça, que pode exercer influência sobre a posição e os movimentos da mandíbula, evidenciando a relação entre o posicionamento da cabeça e os distúrbios temporomandibulares s, $^{1,3,6,8}$.

$$
\text { A relação da articulação }
$$

temporomandibular com as estruturas da cabeça, pescoço e cintura escapular, dificultam - diagnóstico da disfunção craniocervicomandibular e exige do cirurgiãodentista uma avaliação integral do paciente, indo além dos dentes e da boca, afim de propor o melhor plano de tratamento e incluindo também, outros profissionais como fisioterapeutas, fonoaudiólogos e psicólogos. Neste caso, o correto diagnóstico da condição e a localização da sua origem ascendente foi a chave para a escolha da modalidade terapêutica a ser instituída e, consequentemente, para a resolutividade do caso, estando o paciente sem sintomatologia dolorosa, após um mês do início do tratamento ${ }^{12,13}$.

A fisioterapia tem sido uma modalidade de tratamento bastante usada no tratamento de pacientes com disfunções musculares, 
disfunções

temporomandibulares, craniocervicomandibulares e qualquer outras que demandem ou não contiguidade. É classificada como uma terapia de suporte, sendo instituída em conjunto com um tratamento definitivo nas diversas desordens temporomandibulares ou desordens craniocervicomandibulares. A realização de um acompanhamento fisioterapêutico tem resultado em uma melhora na funcionalidade e na mobilidade mandibular e cervical, provocando um mínimo de dor e em curto período de tempo, além disso, tem prevenido recidivas da disfunção $11,14-19$.

Alguns estudos apontam a eficácia dos exercícios posturais associados a terapia manual e acupuntura na redução da dor e melhora da função e abertura bucal. Uma revisão sistemática analisou os estudos que avaliaram a eficácia de várias intervenções fisioterapêuticas na DTM e concluiu que os exercícios ativos, as mobilizações manuais, o treinamento postural em combinação com outras intervenções, incluindo placas estabilizadoras miorrelaxantes, a terapia a laser de baixa intensida, os programas de retroalimentação (biofeedback), relaxamento e reeducação proprioceptiva podem ser mais eficazes do que o tratamento placebo ou que 0 uso isolado de placas miorrelaxantes ${ }^{16,20,21}$.

Neste relato de caso apresentado, a abordagem fisioterapêutica foi 0 primeiro tratamento a ser instituído, sendo escolhido como terapia principal e visando inicialmente o condicionamento e o alongamento da musculatura cervical, para que posteriormente, a musculatura mastigatória pudesse ser trabalhada através de exercícios contra resistência, em associação ao uso de uma placa miorrelaxante. Trabalhos mostram que os exercícios terapêuticos associados à placa miorrelaxante tiveram efeitos significantes no alívio da dor e na disfunção, e em alguns casos, somente o exercício terapêutico foi eficaz, podendo ser recomendado como primeira opção de tratamento na disfunção miogênica ${ }^{16,22}$.

CONCLUSÃO

Disfunções craniocervicomandibulares apresentam relação com o sistema manducatório, logo, o sistema estomatognático é diretamente conectado ao sistema músculoesquelético.

Essa condição evidencia a importância da realização de uma avaliação integral do paciente pelo cirurgião-dentista, examinando-o como um todo, indo além dos dentes e da boca, sendo a postura corporal um dos principais fatores desencadeantes e perpetuantes destas disfunções. Esta avaliação possibilita alcançar um diagnóstico correto e preciso.

O diagnóstico da condição é a chave para a escolha e o sucesso do tratamento. Deve-se optar, primeiramente, pelas terapias conservadoras como a abordagem fisioterápica, que tem mostrado excelentes resultados na resolução dos casos com disfunção craniocervicomandibular, apresentando como benefícios a simplicidade de execução, a utilização de técnicas não invasivas e o baixo custo.

Além disso, o acompanhamento clínico em longo prazo é de extrema importância para a manutenção do quadro do paciente, sem que volte a apresentar os sinais e sintomas característicos da disfunção, e reconhecendo possíveis fatores condicionantes e determinantes para a mesma, como as alterações cervicais.

\section{REFERENCIAS}

1. Rocabado M. Relaciones biomecânicas de las regiones craneales, cervicales e hioideas. Ortodoncia. 1994;58(115):51-6.

2. Barbosa VCS, Barbosa FS. Abordagem fisioterápica. In: Barbosa VCS, Barbosa FS. Fisioterapia nas disfunções temporomandibulares. São Paulo: Phorte; 2009. p.106-190.

3. Amantéa DV, Novaes AP, Campolongo GD, Barros TP. A importância da avaliação postural no paciente com disfunção da articulação temporomandibular. Acta ortop bras. 2004; 12(3):155-59.

4. lunes DH. Análise da postura crânio-cervical em pacientes com disfunção temporomandibular [Tese]. Ribeirão Preto: Faculdade de Medicina de Ribeirão Preto, Universidade de São Paulo; 2007.

5. Cuccia A, Caradonna C. The relationship between the stomatognathic system and body posture. Clinics (Sao Paulo). 2009;64(1):61-6.

6. Gonzalez HE, Manns A. Forward head posture: its structural and functional influence on the stomatognathic system, a conceptual study. Cranio. 1996;14(1):71-80.

7. Weber P, Correa ECR, Ferreira FS, Soares JC, Bolzan GP, Silva AMT. Frequência de sinais e sintomas de disfunção cervical em indivíduos com disfunção temporomandibular. J Soc Bras Fonoaudiol. 2012;24(2):134-39.

8. Nicolakis $P$, Nicolakis $M$, Piehslinger $E$, Ebenbichler G, Vachuda M, Kirtley $C$ et al. Relationship between craniomandibular disorders and poor posture. Cranio. 2000;18(2):106-12.

9. Portero PP, Kern R, Kusma SZ, Grau-Grullón P. Placas oclusais no tratamento da disfunção 
temporomandibular (DTM): revisão de literatura. Rev Gestão \& Saúde. 2009; 1(1):36-40.

10. Barreto DC, Barbosa ARC, Frizzo ACF. Relação entre disfunção temporomandibular e alterações auditivas. Rev CEFAC. 2010; 12(6):1067-1076.

11. Polo V. Avaliação da terapia de aplicação de calor seco em portadores de dor orofacial, disfunção temporomandibular e crânio cervical. [monografia]. Piracicaba, SP: Faculdade de Odontologia de Piracicaba - UNICAMP; 2013.

12. Donnarumma MC, Muzilli CA, Ferreira C, Nemr K. Disfunções temporomandibulares: sinais, sintomas e abordagem multidisciplinar. Rev CEFAC. 2010;12(5):788-94.

13. Borel KC, Silva VCC, Barbosa FS. Prevalência de sinais e sintomas de disfunção temporomandibulares em universitários de Muriaé, MG. Rev Cient FAMINAS. 2008;3(2):61-70.

14. Cerdeira DQ, Nunes TTV, Santiago ASN, Lima AC. Disfunção temporomandibular: a vivência fisioterapêutica na clínica de dor orofacial. Fisioter Bras. 2012;13(2):96-102.

15. Tridapalli ALR. Tratamento Fisioterápico das Desordens Temporomandibulares. [monografia]. Florianópolis: Universidade Federal de Santa Catarina - UFSC; 2012.

16. Maluf AS, Moreno BGD, Alfredo PP, Marques AP, Rodrigues G. Exercícios terapêuticos nas desordens temporomandibulares: uma revisão de literatura. Fisioter Pesq. 2008;15(4):408-15.

17. Tedeschi MF, Marques AP, Marzola C. Contribuição da fisioterapia para a Odontologia nas disfunções da articulação temporomandibular. Rev odonto ciênc. 2002; 17(36):119-34.

18. Grade R, Caramês J, Pragosa A, Carvalhão J, Sousa S. Postura e disfunção temporomandibular: controvérsias atuais. Rev Port Estomatol Cir Maxilofac. 2008; 49(2):111-17.

19. Okeson JP. Considerações gerais no tratamento das desordens temporomandibulares. In: Okeson JP. Tratamento das desordens temporomandibulares e oclusão. 6. Ed. Rio de Janeiro: Elsevier; 2008. p. 269-301.

20. McNeely ML, Armijo OS, Magee DJ. A systematic review of the effectiveness of physical therapy interventions for temporomandibular disorders. Phys Ther. 2006; 86(5):710-25.

21. Medlicott MS, Harris SR. A systematic review of the effectiveness of exercise, manual therapy, electrotherapy, relaxation training, and biofeedback in the management of temporomandibular disorder. Phys Ther. 2006;86(7):955-73.
22. McNamara JA, Seligman DA, Okeson JP. Occlusion, orthodontic treatment, and temporomandibular disorders: a review. J Orofac Pain. 1995;9(1):73-90.

\section{CONFLITO DE INTERESSES}

Os autores declaram não haver conflitos de interesse

\section{AUTOR PARA CORRESPONDÊNCIA}

\section{Bárbara Nathalia Marques de Lima}

Rua Santiago Dantas, no 04, Bairro Novo Israel, 69039-090Manaus - AM, Brasil

Telefone: (92) 98468-2571

Email: barbara.nml@gmail.com
Submetido em 12/12/2019 Aceito em 22/10/2020 